\title{
RECORRIDO POR LA IMAGEN SOCIAL DE LA FORMACIÓN PROFESIONAL: UN CAMINO HACIA SU REVALORIZACIÓN
}

An overview of the social image of Vocational

Education: A path towards its appreciation

\author{
Enrique García Jiménez* y Rocío Lorente García**
}

\section{RESUMEN}

Históricamente, la formación profesional en España ha sufrido una situación de descuido político que, a diferencia de otros países industrializados, le ha otorgado un desprestigio social que queda reflejado en los menores niveles de matriculación en estas enseñanzas, tradicionalmente consideradas "de segunda clase", con respecto a los estudios universitarios. Si bien es cierto, se han desarrollado en las últimas décadas iniciativas políticas orientadas a prestigiar la formación profesional. Señalaremos, por tanto, cuáles son estas iniciativas y características propias de estas enseñanzas que, junto a la actual situación económica y social han ayudado a transformar positivamente su imagen social y, por ende, han contribuido a un significativo aumento de matriculados. Asimismo, en este artículo se presentan los resultados de una investigación que permiten vislumbrar que esta evolución de la imagen social de la formación profesional es percibida por el propio profesorado que, como veremos, considera que se ha avanzado positivamente en la dignificación de estas enseñanzas.

\footnotetext{
* Centro de Magisterio "La Inmaculada", Universidad de Granada (España).

${ }^{* * *}$ Universidad de Jaén (España).
} 
PALABRAS CLAVE: Formación Profesional, Percepción Social, Política Educativa.

\begin{abstract}
Historically, Vocational Education in Spain has suffered a situation of political oversight that, unlike other industrialized countries, it has been conferred a social discredit that is reflected in the lower levels of enrollment in these teachings, traditionally considered "second class" in comparison to university studies. While it is true, it has been developed in recent decades political initiatives to prestige Vocational Education. We point out, hence, what these initiatives are and characteristics of these teachings which, together with the current economic and social situation have helped to positively transform their social image and, therefore, have contributed to a significant increase in enrollment. Also, in this paper the results of an investigation is presented that allow to think that this evolution of the social image of Vocational Education is perceived by teachers that, as we shall see, consider that it has made good progress in the dignity of these teachings.
\end{abstract}

KEY WORDS: Vocational Educaiton, Social Perception, Educational Policy.

$* * * * * *$

\title{
1. INTRODUCCIÓN
}

Históricamente, la formación profesional (en adelante FP) en España ha sufrido una situación de descuido político que, a diferencia de otros países industrializados, le ha otorgado un desprestigio social que queda reflejado en los menores niveles de matriculación en estas enseñanzas, consideradas "de segunda clase", con respecto a los estudios universitarios.

En este artículo, para entender a qué responde este desprestigio, haremos un breve recorrido por la historia político-normativa de la formación profesional en nuestro país, centrándonos en aquella normativa que, en su momento, y durante varias décadas ha mantenido a estas enseñanzas profesionales desvalorizadas.

Si bien es cierto, se han desarrollado en las últimas décadas iniciativas políticas orientadas a prestigiar la formación profesional. Señalaremos, por tanto, cuáles son estas iniciativas y características propias de estas enseñanzas que han ayudado a transformar positivamente su imagen social y, por ende, han contribuido a un significativo aumento de matriculados.

Esta evolución de la imagen social de la formación profesional es percibida por el propio profesorado que, como veremos, sostiene que si bien la formación profesional ha 
tenido una consideración negativa en el pasado, se ha avanzado positivamente en la dignificación de estas enseñanzas. De hecho, las cifras así lo demuestran.

El actual contexto social y económico en España, marcado por la existencia de una fuerte crisis, cuyas consecuencias más nefastas son el paro y la precariedad laboral, puede estar ayudando también a dar un vuelco a la situación de los estudios de formación profesional, junto con estas iniciativas políticas señaladas, incidiendo en una mayor matriculación, como sostenemos a lo largo del artículo. De esta forma, tanto el marco normativo como el contexto social y económico estarían facilitando un cambio de percepción sobre la formación profesional, redundando en una mejor imagen social.

No obstante, no podemos negar que todavía existen algunos resquicios de este desprestigio, manifestados en el mayor número de matriculados en estudios universitarios, por lo que aún puede hacerse mucho más a nivel institucional y desde los poderes públicos para continuar el camino emprendido con la finalidad de prestigiar las enseñanzas de Formación Profesional.

\section{UN ANTES Y UN DESPUÉS DE LA IMAGEN SOCIAL DE LA FORMACIÓN PROFESIONAL}

\section{$\underline{\text { 2.1. El desprestigio de la Formación Profesional }}$}

A pesar de las transformaciones políticas que, como veremos, se han producido en materia de FP, encaminadas a la mejora de ésta y a su ajuste con la realidad económica y social, históricamente ha planeado sobre ella una imagen negativa que ha repercutido sustantivamente en los matriculados en FP en relación con otros estudios.

Apoyando esta afirmación con cifras, según un estudio del Instituto de la empresa familiar publicado en 2008, "en 1990 el 65\% de los estudiantes que decidían continuar con su educación tras completar la educación secundaria obligatoria elegían hacerlo en el bachillerato. Además, la mayor parte de este porcentaje no había elegido la FP como opción preferida, sino que se veía empujada a ella por unos malos resultados escolares" (QUINTANA, 2008: 26). Si bien estos datos de matriculación han ido evolucionando hacia el aumento en la FP, encontramos aún que las diferencias de acceso, con respecto al resto de enseñanzas están aún muy marcadas. "De hecho, en el periodo 2000/2005 el incremento de alumnos de FP, tanto de grado medio como de grado superior, fue superior al $20 \%$ pero, aún así, España sigue alejada de la media de OCDE, en la que bachillerato y FP reciben la misma proporción de alumnos, el 50\%" (QUINTANA, 2008: 26). 
Para comprender mejor a qué responde este desprestigio que ha influido tanto en las diferencias de acceso de la FP con respecto a demás estudios (bachillerato y universitario) hay que remitirse a la historia de la política educativa referente a la FP en nuestro país.

La institucionalización definitiva de las enseñanzas profesionales comenzó en la década de 1870, prolongándose hasta el siglo XX. Será con la Ley Orgánica de Formación Profesional Industrial (1955), cuando se inicia el proceso de consolidación de las enseñanzas profesionales. Con esta ley, estas enseñanzas se estructuraban en Preaprendizaje, Aprendizaje y Maestría. Pero será en 1970, con la Ley General de Educación y Financiamiento de la Reforma Educativa (promovida por Villar Palasí), cuando se produzca un giro político sustantivo a la FP, otorgándole un carácter educativo.

Mediante esta ley, el sistema se reestructura en una etapa de Educación General Básica (EGB) que termina a los 14 años y que se establece único, obligatorio y gratuito para todos los españoles. Desde la EGB se accedía Bachillerato Unificado Polivalente (BUP), denominado así por ofrecer una amplia diversidad de experiencias prácticoprofesionales, permite el mejor aprovechamiento de las aptitudes de los alumnos y evitar el carácter excesivamente teórico y academicista que lo caracterizaba, y que tenía una duración de tres años (15, 16 y 17 años). Desde el BUP se accedía, a su vez, al Curso de Orientación Universitaria (COU), o en su defecto a la FP. La citada ley, señala como finalidad específica de la FP la capacitación de los alumnos para el ejercicio de la profesión elegida, además de continuar su formación integral. Deberá guardar, en su organización y rendimiento, estrecha relación con la estructura y previsiones del empleo. Para ello, concretamente, organizaba la FP en tres niveles: Iniciación o primer grado (FPI); Nivel Medio o segundo grado (FPII); y de Nivel Superior o tercer grado. En cuanto al acceso a dichos niveles, la ley especificaba (en su capítulo III) que deberán acceder a los estudios y prácticas de la Formación Profesional de Primer Grado quienes hayan completado los estudios de la Educación General Básica y no prosigan estudios de Bachillerato; a la Formación Profesional de Segundo Grado quienes posean el título de bachiller y quienes, habiendo concluido la Formación Profesional de primer grado, sigan las enseñanzas complementarias que sean precisas, de las que podrán ser dispensados aquellos que demuestren la debida madurez profesional; y a la Formación Profesional de tercer grado, además del alumnado que haya concluido el primer ciclo de una Facultad o Escuela Técnica Superior, todos los graduados universitarios y los de Formación Profesional de segundo grado que hayan seguido las enseñanzas complementarias correspondientes.

Con esta ley se garantizaba que en cualquier momento del proceso educativo, pasado el período de Educación General Básica, se ofrecen al alumno posibilidades de FP, así como la reincorporación a los estudios en cualquier época de su vida de trabajo. Nos encontramos con que por primera vez, la FP y técnica está presente en el conjunto del sistema escolar pero no consigue integrarla en el mismo. Al contrario, la FP parecía 
destinada a los estudiantes fracasados del sistema. De esta manera, la reforma de la FP en la LGE de 1970 supuso un cambio sustancial con respecto a su presencia en el conjunto del sistema escolar, pero lo convierte en un período poco afortunado para estos estudios y no por la ley en sí, que en sus objetivos la potenciaban, sino porque los decretos que la desarrollaron la convirtieron en una vía de segunda clase (paralela al bachillerato y como una sección desvalorizada reflejo institucional de la división social del trabajo) refugio para el fracaso escolar (DE PABLO, 1997; CASQUERO, 2006).

Las repercusiones de la normativa que regula la educación durante tres décadas, se traduce en unas tasas de matriculación en FP muy bajas con respecto a otros estudios. Una de las consecuencias de esto la desarrolla Homs (2008: 128) que sostiene que "el modelo de cualificación español, a diferencia de Europa, que presenta un sistema productivo que se asienta sobre una base de cualificaciones intermedias, en España se basa en una combinación de cualificaciones bajas y superiores". Este exceso de cualificaciones superiores en España frente al déficit que nos encontramos de titulados medios, conlleva a su vez un problema de "sobrecualificación" en el sentido de que "las empresas han utilizado mano de obra con titulaciones superiores (que es la abundante), en lugar de exigir más oferta de cualificaciones intermedias; esto ha dado lugar a un modelo de cualificación poco eficiente y con un resultado de baja productividad" (HOMS, 2008: 129).

\section{$\underline{\text { 2.2. Hacia la revalorización de la Formación Profesional }}$}

Después de la Ley de educación de 1970, será la Ley de Ordenación General del Sistema Educativo (llamada por sus siglas LOGSE), promulgada en 1990, la que supondrá la completa reestructuración del sistema de enseñanza y de FP inicial en nuestro país.

Concretamente, esta ley de educación, con respecto a la FP, hace una distinción entre la FP de base o formación básica de carácter profesional (artículo 30.3) y la FP específica. Al referirse a la FP específica, la define como el conjunto de conocimientos, aptitudes y capacidades relativas a una profesión, es decir, a la competencia para ejercer una serie de puestos de trabajo afines a un campo profesional. La FP específica facilitará la incorporación de los jóvenes a la vida activa, contribuirá a la formación permanente de los ciudadanos y atenderá a las demandas de cualificación del sistema productivo (artículo 30.5). Por tanto, la FP específica se sitúa entre la FP de base y la formación en el puesto de trabajo, sirviendo de puente entre la escuela y la actividad profesional.

Otro de los cambios de esta ley para con la FP específica y, a fin de hacerla más cercana a la realidad laboral, es la estructuración de ésta en ciclos formativos con organización modular, constituidos por áreas de conocimiento teórico-prácticas en función de los diversos campos profesionales, pudiendo acceder a ellas quienes se hallen en posesión del título de Graduado en Educación Secundaria, para poder cursar los ciclos de 
grado medio y, quienes estén en posesión del título de bachiller, para acceder a los ciclos de grado superior.

Por tanto, y al margen de la retórica de "redención social" que acompañó a la reforma socialista de los años noventa (PEREYRA, GONZÁLEZ FARACO, LUZÓN y TORRES, 2009), sus modificaciones en cuanto a los requisitos de acceso a la FP específica suponen una importante iniciativa en cuanto a la dignificación y revalorización social de la FP. Hasta el momento, la existencia de una doble titulación al final de la Educación General Básica, resultaba discriminatoria, ya que posibilitaba el acceso a la FP a quienes no finalizaban positivamente la EGB, convirtiendo a la FP, como ya hemos aludido anteriormente, en una alternativa subsidiaria o estudios de segunda clase.

Asimismo, la LOGSE, en lo referente a la FP, supuso un profundo cambio, diseñando unos estudios profesionales renovados, flexibles, adaptados al mercado laborar y homologables con los restantes países de la Unión Europea. En este sentido, Pérez Esparrells (2001: 102) sostiene sobre esta ley que "puede ser considerada como el primer antecedente del Sistema Nacional de Cualificaciones ya que, como hemos desarrollado, en ella se concibe la FP como un sistema en el que han de integrarse los diferentes subsistemas. Además, formula un replanteamiento de la formación reglada/inicial con la finalidad de conectarla con la formación ocupacional/continua y, por ende, con el mercado de trabajo, a través del gran avance que supone la estructura modular y el incremento de los niveles de cualificación con respecto al antiguo sistema". Será en el marco de esta ley cuando se pongan en marcha numerosas iniciativas políticas encaminadas a la redefinición de la FP para acrecentar el valor de ésta.

Muestra de esto es la aprobación, en 1998, del II Programa Nacional de Formación Profesional $^{1}$, considerado el promotor de la integración de los tres subsistemas de FP (reglada, ocupacional y continua). Con esta iniciativa, se pretende acercar ambos sistemas, el educativo y el productivo, buscando la adecuación de las cualificaciones profesionales que proporcionan cada uno de los subsistemas de FP con las competencias profesionales que, en cada momento, demande el sistema productivo.

No olvidemos que este programa se apoya en la idea, promovida por la Unión Europea, de aprendizaje a lo largo de la vida ${ }^{2}$ y mejora de la calidad de las enseñanzas profesionales. Para conseguir ambos objetivos se crea el Sistema Nacional de Cualificaciones Profesionales, instrumento diseñado para promover y desarrollar la integración de las ofertas de la FP mediante un Catálogo Nacional de Cualificaciones

\footnotetext{
${ }^{1}$ El I Programa Nacional de FP se aprobó en 1985, gestionado por la administración laboral y orientado al impulso de la formación de los trabajadores (formación ocupacional).

${ }^{2}$ Esta idea de aprendizaje a lo largo de la vida, no dejará de estar presente en las posteriores iniciativas (y hasta nuestros días), siendo un aspecto clave en la FP.
} 
Profesionales. Asimismo, busca promover y desarrollar la evaluación y acreditación de las correspondientes competencias profesionales, de forma que se favorezca el desarrollo profesional y social de las personas y se cubran las necesidades del sistema productivo.

Y si hablamos de las iniciativas llevadas a cabo en nuestro país para cambiar la negativa imagen que ha caracterizado a la FP, se debe hacer especial mención a la Ley Orgánica de las Cualificaciones y de la Formación Profesional, (LOCFP), aprobada en 2002, pues será la responsable de materializar las iniciativas y propuestas planteadas en el I y II programas de FP.

Concretamente, esta ley será la responsable de la actual vinculación de la FP del sistema educativo y la FP para el empleo, despareciendo los tres subsistemas tradicionalmente existentes. A partir de la LOCFP, y los decretos que la desarrollan, se configura un nuevo subsistema que exige la coordinación de organismos y sujetos para el desarrollo de las acciones formativas a que refiere la LOCFP, integrando las ya utilizadas en el ámbito de los subsistemas de FP ocupacional y continua. Por tanto, esta Ley responde a la necesidad encaminada a promover la movilidad de estudiantes y trabajadores en el territorio europeo, objetivo que queda clara en la exposición de motivos, precisa que es a través del "reconocimiento y la acreditación de las cualificaciones profesionales a nivel nacional, como mecanismo favorecedor de la homogeneización, a nivel europeo, de los niveles de formación y acreditación profesional de cara al libre movimiento de los trabajadores y de los profesionales en el ámbito del mercado que supone la Comunidad Europea".

La nueva ley Orgánica de Educación que, en 2006) sustituye a la LOGSE (conocida como LOE), defenderá una FP en los mismos términos que lo hace la LOCFP. Otra de las novedades que supone la LOCFP, en la línea de conectar los sistemas educativo y productivo y que será un aspecto clave en la renovación de la imagen de la FP es la propuesta de una doble vía para la adquisición de competencias profesionales.

Una de estas vías es la experiencia laboral. Esto es, el reconocimiento de las competencias profesionales adquiridas a través del desempeño de una labor profesional. Esto requiere una compleja labor de organización, estructuración y negociación de procedimientos o técnicas para determinar qué experiencia laboral se exige para considerar que se ha adquirido esa competencia en concreto, a través de qué actividades desarrolladas y en qué contexto ${ }^{3}$.

\footnotetext{
${ }^{3}$ A partir de junio de 2011, se puso en práctica en las distintas Comunidades Autónomas la posibilidad de la acreditación de competencias profesionales. Para más información: http://www.educacion.gob.es/educa/incual/ice_recAcr.html (último acceso el 22 de octubre de 2014).
} 
La segunda de las vías de adquisición de competencias es la formativa. Cabe la posibilidad de que una persona no posea competencia profesional alguna porque nunca ha desempeñado ningún trabajo, en cuyo caso podrá adquirirla participando en procesos de formación con efectos en el empleo. También es posible que una persona tenga conocimientos suficientes para realizar unas determinadas funciones, (porque lleve muchos años desempeñando una actividad laboral) $\mathrm{y}$, aunque esta persona podría considerarse competente, ello no tiene por qué implicar que esa persona esté cualificada, ya que la cualificación se refiere a suma de competencias. Si dicha persona quisiera estar cualificada (lo que le permitiría cambiar de trabajo, de país, de actividad a desarrollar, ascender, etc.), podría necesitar la adquisición de una serie de destrezas adicionales, que podría adquirir a través del trabajo. Igualmente puede adquirir nuevas competencias por la participación en procesos de formación ${ }^{4}$.

Pero el cambio más obvio es que se establece una nueva estructura de la FP impartida dentro del sistema educativo que permite flexibilizar la FP. La nueva estructura comprende un conjunto de ciclos formativos con una organización modular, de duración variable y contenidos teórico-prácticos adecuados a los diversos campos profesionales. Por tanto, los ciclos formativos son de grado medio y de grado superior, y están referidos al Catálogo Nacional de Cualificaciones Profesionales y constituyen, respectivamente, la FP de grado medio y la FP de grado superior. El currículo de estas enseñanzas se ajustará a las exigencias derivadas del Sistema Nacional de Cualificaciones y Formación Profesional (SNCFP). Dicha estructura modular de los ciclos, permite la inscripción para realizar módulos y no sólo cursos completos, otro aporte fundamental para la flexibilización del sistema.

Dentro de la nueva estructuración de la FP, se contempla la formación en centros de trabajos, lo que supone establecer vías de comunicación y relaciones entre los centros formativos y las empresas. Esta formación en empresas permite una conexión con la realidad laboral, que va a ayudar a la potenciación de la inserción laboral de alumnado y, por ende, a la imagen social de la FP.

Esta acreditación de competencias adquiridas tanto en el laboral como formativo responde a las directrices establecidas en la Unión europea en 2004, sobre Principios comunes europeos para la validación del aprendizaje no formal e informal, necesarios para fomentar la comparabilidad, la transferencia y aceptación de los resultados educativos y formativos.

La introducción de estos elementos de flexibilidad y facilidades de acceso entre los diferentes niveles educativos, entre la FP y el mercado de trabajo y entre las enseñanzas

\footnotetext{
${ }^{4}$ Esta formación siempre tendrá que tener como referente el Catálogo Nacional de las Cualificaciones Profesionales.
} 
generales y las profesionales "ha significado una importante renovación de la estructura básica de la FP, manteniendo su coherencia y simplicidad” (HOMS, 2008: 44).

\section{UNA RENOVADA CONCEPCIÓN DE LA FP: PRECEPCIÓN DEL PROFESORADO}

Sabiendo que este es un tema del que se ha hablado y escrito mucho, queríamos saber la opinión del profesorado de FP al respecto ${ }^{5}$, pues resulta interesante conocer la visión de aquellos actores sociales que están implicados en la misma, pues "a menudo cuando se aplican reformas educativas se privilegian datos cuantitativos respecto a la matrícula o al rendimiento, pero son pocos los balances que se hacen de las reformas a partir de las voces de quienes las están protagonizando" (MERINO, 2003: 5-6).

Concretamente, al abordar con el profesorado de FP el tema de su imagen social se detecta que existe un pensamiento generalizado de que históricamente estos estudios han estado muy poco valorados y cuando habla en tiempo pasado sobre la imagen de la FP, lo hace reseñando el desprestigio que ha acompañado a estas enseñanzas.

En algunos casos, se utilizan atributos muy concretos que definen claramente cómo la FP ha sido considerada en el pasado, en comparación a las demás enseñanzas del sistema educativo como "hermana pobre del sistema" o "la cenicienta".

“Al comienzo era la hermana pobre de la educación." (Profesor 2)

"El que venía aquí era el que no servía para otra cosa." (Profesor 1)

Vemos que, principalmente identifican esta enseñanza como "remedial", considerada como la enseñanza a la que iban los "torpes", los "malos", los "fracasados" de la educación, los que no querían estudiar y buscaban como solución la inserción en la vida laboral. En otros casos, si bien los entrevistados no hacen referencia explícita a la imagen social de la FP antes de la implantación de la LOGSE, al hablar de la imagen actual de ésta dejan latente que tradicionalmente no ha sido positiva.

"La imagen de la FP siempre ha sido un poco bueno, el que no puede hacer el Bachillerato, el que no puede hacer una carrera universitaria, pues que se vaya a la Formación Profesional." (Profesora 29)

Esta imagen social que ha caracterizado a la FP a lo largo de decenios se ha arrastrado hasta nuestros días. En este sentido algunos profesores hablan de "lacra",

\footnotetext{
${ }^{5}$ Los resultados que aquí se presentan, son parte de una investigación más amplia centrada en el analizar la evolución de las reformas de FP en España. Esta investigación, de carácter cualitativa, fue desarrollada entre los años 2008 y 2010, y se centró en el análisis del discurso extraído de entrevistas abiertas a 50 profesores y profesoras de centros educativos donde se imparte FP en las ocho provincias de la región andaluza. Distintas partes del mismo estudio ya han sido abordadas y publicadas en otros trabajos.
} 
"fama", "lastre", como apelativos y atributos que aluden al desprestigio social que tradicionalmente ha sufrido la FP y, reflejan que, de algún modo, aún hoy sigue pesando sobre ella.

Aunque en el discurso se aprecia que hoy está más valorada que en el pasado, el profesorado se queja de que aún hoy recaen sobre ella antiguos estereotipos.

En el discurso, parte del profesorado alude al propio sistema, que concibe como vía de "segundo orden" o vía "alternativa" los ciclos formativos, participando de esta imagen. Así, orientadores, profesorado de secundaria,... todavía hoy, animan al alumnado desmotivado a cursar algún ciclo formativo. Por el contrario, al alumnado que va bien en sus estudios se les empuja hacia estudios universitarios, sin tener otros factores en cuenta.

"Hoy en día hay mucho profesorado en secundaria que desvía a los alumnos a los ciclos formativos a los alumnos fracasados, pero sí con menos rendimiento, eso es una realidad total.” (Profesor 44)

Además, habría que considerar igualmente, la idea socialmente extendida (y equivocada según el profesorado entrevistado) de que los ciclos formativos presentan menos dificultad académica por su vinculación con el mundo laboral y su perfil práctico, y por consiguiente son más fáciles de superar. Esta sensación de devaluación del conocimiento técnico, más pragmático, también se percibe en el discurso de muchos de los profesores, como una "minuvaloración” de su trabajo.

Relacionado con esta percepción negativa de las enseñanzas profesionales por parte de alumnado, profesorado y familias; las bajas tasas de matriculación con respecto a otras enseñanzas; el profesorado ineludiblemente la contrasta con la valoración que en otros países se tiene de estas enseñanzas. Una aportación recurrente para buscar alternativas, así como la inclusión de España en la Unión europea, también ha supuesto avances considerables en materia de $\mathrm{FP}^{6}$ que, a su vez, pueden servir para prestigiarla y dotarla de contenido. Sin embargo, un aspecto que sobresale como positivo entre los docentes entrevistados es el hecho de que se hayan cambiado los requisitos de acceso a la FP, lo que puede favorecer la imagen de ésta.

"Siempre la gente que no servían para bachillerato se iban a FP, eso era antes, ahora como a la FP se le exige el bachillerato pues parece que eso ha servido, por lo cual yo creo que esa es una buena idea." (Profesor 20)

\footnotetext{
${ }^{6}$ Así por ejemplo, siguiendo las directrices de la Unión Europea y para favorecer la libre circulación de trabajadores por el territorio comunitario, se plantea la exigencia de facilitar la transparencia de calificaciones en el ámbito comunitario, a lo que también se hace referencia en el discurso del profesorado, y hemos analizado en el capítulo anterior.
} 
En esta línea, cabe destacar que en algunos casos, al hablar sobre la imagen social que existe sobre la FP, hay profesorado que opina que habría que hacer una diferenciación entre las enseñanzas profesionales de Grado Medio (GM) y de Grado Superior (GS), pues los ciclos formativos de GS están bastante mejor valorados que los de GM, por el entorno social y también económico. Para otro sector del profesorado, la distinción la hacen, no tanto entre los ciclos de GS y de GM, como entre Familias Profesionales, reseñando que hay unas más desprestigiadas que otras (haciendo mención a la clásica distinción entre las familias profesionales de "bata" y "mono"), en alusión a la categorización clásica en la sociología de las profesiones.

"Pero la FP que sea una formación de $2^{a}$ está en el país, esto es normal, nadie quiere ponerse un mono, había formaciones profesionales de bata blanca y de mono, y las de mono estaban desprestigiadas, ahora ya alguna de mono, nosotros tenemos las clásicas de mecánica, o del automóvil, pues ya están un poquito más prestigiados, pero la gente no quiere ponerse el mono." (Profesor 37)

No obstante, y a pesar de las subjetividades y el pesimismo en torno a la imagen de la FP, sigue existiendo una gran mayoría de profesorado que considera y valora su evolución, a pesar de los desencuentros y desajustes que aún persisten, sobre todo por parte del profesorado de otras enseñanzas y de las familias que no la conocen. Esta apreciación recoge la dignificación que representa este nivel educativo respecto a etapas educativas anteriores. Este hecho se ha observado en numerosos fragmentos, por ejemplo:

"Pues vamos a ver, como todo tiene sus puntos flacos y sus puntos fuertes, evidentemente. Entonces para mí hay una cosa importante, que desde que se produce ese cambio, la FP se equipara al resto de la enseñanza." (Profesora 25)

Pero frente a un discurso mayoritario que valora positivamente los cambios acaecidos en las últimas décadas, encontramos una pequeño índice de casos en los que persiste la imagen nostálgica del modelo anterior a 1990, que se corresponde con un modelo más escolar ${ }^{7}$.

\section{IMAGEN DE LA FP EN EL CONTEXTO ECONÓMICO Y SOCIAL ACTUAL}

Actualmente, y desde el año 2008, nos encontramos en una España marcada por una profunda crisis económica, que ha provocado a su vez una gran inestabilidad social en todas sus vertientes, pero especialmente en lo referente al aspecto laboral y al educativoformativo.

Respecto al ámbito laboral, la mayor consecuencia de la crisis se ve reflejada en los niveles dramáticos de desempleo existentes actualmente en España. En este sentido, al

\footnotetext{
${ }^{7}$ Concretamente, esta valoración negativa sobre la evolución de la FP, es manifiesta en 8 casos de un total de 50.
} 
cierre del mes de septiembre de 2014, las cifras oficiales de paro en España señalan la existencia de 4.447.650 personas en situación de desempleo, según datos del Ministerio de Empleo $^{8}$, si bien es cierto que en los últimos doce meses, desde septiembre de 2013, la cifra de parados ha descendido en 276.705 personas, debido a los indicios macroeconómicos de cierta recuperación. Asimismo, la tasa de paro en España según la Encuesta de Población Activa (EPA) en el tercer trimestre de 2014 es del 23,67\%, habiendo llegado en el cuarto trimestre de 2012 al nivel más elevado, con un $26,9 \%$.

Además del desempleo, otra problemática es la gran precariedad laboral, abundando contratos temporales, de prácticas, de formación, por debajo de la cualificación obtenida, etc. Son los conocidos como "contratos basura".

Todo esto guarda relación con el trabajo flexibilizado y/o trabajo devaluado, "al pasar de un modelo de empleo estable y homogéneo -fundamentado en un trabajo fijo, estable y con posibilidades reales de ascenso laboral, y con condiciones laborales regularizadas para la mayoría de los trabajadores- a otro mucho más inestable y heterogéneo, con mayoría de contratos de trabajo atípicos y precarios" (Rubio, 2009:59).

Por otra parte, respecto al ámbito educativo- formativo, la inestabilidad viene derivada de dos vertientes. Por un lado, la problemática que conlleva la realización de un importante esfuerzo familiar para cursar una enseñanza universitaria, teniendo en cuenta la coyuntura económica y las dificultades reales de muchas familias actualmente, unido a la política de recortes y restricciones respecto al Estado de bienestar (que se manifiestan en el campo educativo en medidas como la reducción de becas, el aumento de tasas universitarias, etc.). Por otro lado, la falta de seguridad laboral en cuanto a la consecución de un empleo acorde con la cualificación de la enseñanza universitaria cursada en una condiciones óptimas (esto es, fuera del rango de la inestabilidad y precariedad laboral) dadas las características actuales del mercado laboral español, apuntadas brevemente con anterioridad en lo referente al problema del desempleo y de la precariedad laboral.

Ante tal panorama, toman especial protagonismo los estudios de FP. En este sentido, los datos en cuanto a matriculación, en constante ascenso, son una clara muestra de que la FP en nuestro país empieza a ser valorada social y económicamente. De hecho, el alumnado matriculado en estas enseñanzas en España crece constantemente desde el inicio de la crisis en el año 2008, tanto en Grado Medio como en Grado Superior. En este sentido, en el curso académico 2007/2008 el número de alumnos y alumnas de FP fue de 462.492, mientras que en el presente curso 2014/2015 ha sido de 793.034, habiéndose producido una

\footnotetext{
${ }^{8}$ Disponible en http://prensa.empleo.gob.es/WebPrensa/noticias/laboral/detalle/2320

${ }^{9}$ Disponible en http://www.ine.es/daco/daco42/daco4211/epa0114.pdf
} 
constante y progresiva evolución positiva en los años intermedios, según datos oficiales del Ministerio de Educación, Cultura y Deporte ${ }^{10}$.

Así pues, y a tenor de estos resultados, parece superada ya esa imagen social negativa que ha perseguido a la FP desde hace décadas. ¿Cómo podemos conectar la realidad actual de los estudios de FP (con el significativo aumento en sus tasas de matriculación) con el contexto social y económico de la España de hoy?

En primer lugar, como queda expuesto más arriba, los propios profesores de FP en las entrevistas realizadas, no dudan en afirmar la mayor conexión de estos estudios con el mercado laboral. Esto es, son considerados estudios más prácticos y más cercanos a la realidad laboral. Desde esta visión, se puede explicar el hecho de que ante una situación laboral tan problemática como la actual, con el paro y la precariedad laboral como grandes exponentes de las consecuencias nefastas de la crisis económica, muchos jóvenes y sus familias piensen en la posibilidad real de acceder a estudios de FP para poder facilitar todo lo posible su ingreso futuro en el mercado laboral. Si existe mayor conexión entre la FP y el mercado de trabajo puede resultar más fácil integrarse en él, a pesar de las enormes dificultades de inserción laboral que tienen los jóvenes actualmente en España, que encuentran un ejemplo muy gráfico en el siguiente dato: en 2012, año culmen de la crisis, la tasa de desempleo de los jóvenes de 16 a 30 años se sitúa en el 38,6\%, frente al 24,6\% de la población total, según datos de la Encuesta de Población Activa en el primer trimestre de $2013^{11}$.

En segundo lugar, tal como mencionamos con anterioridad, muchas familias se encuentran en una coyuntura económica muy delicada, debilitándose sobremanera sus recursos económicos y su capacidad de consumo a todos los niveles. En este sentido, también la opción de cursar una enseñanza universitaria supone un proceso de consumo en cierta medida para la familia, pues el acceso a la universidad conlleva pagar una matrícula (cada vez más elevada), material didáctico y pedagógico y, en algunos casos, una vivienda y gastos manutención y transporte. Sumando a esto que las enseñanzas universitarias son de mayor duración que las enseñanzas técnicas. En muchos casos existe total imposibilidad por parte de las familias de hacer frente a esta serie de gastos. En otros casos sería posible con un enorme esfuerzo equivalente a una serie de renuncias en cuanto a bienestar. Quizás tanto en unos casos como en otros, la alternativa de la FP toma cuerpo como opción real para continuar el proceso formativo de los hijos, que conlleva muchos menos gastos a todos los niveles.

10 Disponible en http://www.todofp.es/todofp/sobre-fp/informacion-general/sistema-educativo-fp/nuevafp.html

${ }^{11}$ Disponible en http://www.ine.es/daco/daco42/daco4211/epa0113.pdf. 
En tercer lugar, siguiendo en la línea de la situación de inestabilidad y heterogeneidad del mercado laboral español, con una gran cantidad de contratos distintos unos de otros, pero muchos de ellos coincidentes en su fuerte componente de precariedad, en la práctica implica que muchos titulados universitarios se encuentran trabajando en empleos por debajo de su cualificación. En concreto, según el Informe 2013 de la Fundación Conocimiento y Desarrollo, el 36\% de los titulados universitarios españoles se encuentran sobrecualificados. Esta cifra es significativamente superior a la de Europa, y además la tendencia sigue un patrón al alza. Ante tal panorama muchos jóvenes y sus familias pueden acudir a la FP con el propósito de ahorrar gastos y acortar el período formativo, ya que el acceso a un puesto de trabajo de alta cualificación se antoja complicado, al tiempo que los estudios de FP se encuentran conectados en mayor medida a la realidad laboral, al ser eminentemente más prácticos. No obstante, esta tesis se tambalea al apreciar que todos los datos al respecto señalan que los titulados universitarios son los que más facilidad tienen para encontrar empleo. Por lo tanto, se encuentran en una situación de sobrecualificación en su puesto de trabajo pero acceden con mayor facilidad al mercado laboral. Según corrobora el propio informe 2013 de la Fundación Conocimiento y Desarrollo, en comparación con las personas que tienen un nivel educativo inferior, los graduados universitarios gozan de una mayor tasa de actividad, mayor tasa de ocupación, mayores ingresos salariales y menor tasa de paro.

\section{CONCLUSIONES}

Atendiendo al discurso del profesorado de FP, podemos señalar que, si bien la FP ha tenido una consideración negativa en el pasado -aunque se ha avanzado positivamente en la dignificación de las mismas- aún hoy existen resquicios de ese desprestigio y se recibe un alto porcentaje de alumnado que ha fracasado en sus estudios y escoge esta opción con el fin de obtener un título que facilite su inserción laboral. No obstante, se extrae de la entrevistas que la FP es una formación muy valorada por quienes la conocen y están en contacto con ella. Así, los empresarios cada vez demandan mayor número de alumnos egresados de los ciclos formativos, siendo su tasa de inserción más alta que la de otras enseñanzas, lo que a su vez, contribuye también a que empiece a estar mejor vista por el alumnado y sus familias y, sobre todo, aumente el número de matriculados.

Por otra parte, no sabemos si a causa del contexto actual de crisis y todas sus connotaciones o como consecuencia del mismo, la imagen social de los estudios de FP ha mejorado ostensiblemente en los últimos años. Poco a poco se va superando esa percepción negativa que vinculaba la realización de estudios de FP con el fracaso escolar y con una posición de segundo orden en la jerarquía educativa. Sea a causa de todo lo señalado o como consecuencia de ello, los estudios de FP son considerados hoy día por numerosos jóvenes y sus familias como una alternativa muy válida y como una opción real para la culminación de su proceso formativo, o incluso como paso previo a los estudios 
universitarios si se da el caso y las circunstancias acompañan. Esta tesis se apoya en la gran evolución positiva de matriculación en estudios de FP en los últimos años en España, cuyos datos han sido expuestos con anterioridad.

No obstante, el porcentaje de matriculados en FP aún es bastante inferior con respecto a las enseñanzas universitarias. En este sentido, el número de matriculados en enseñanzas universitarias en el curso 2013/2014, según datos del Ministerio de Educación, Cultura y Deporte, es de 1.438.115 estudiantes, por 696.648 matriculados en estos estudios en el mismo curso académico. Esto es, algo más del doble de estudiantes universitarios, si bien las diferencias tienden a disminuir año tras año.

A la luz de estos datos, consideramos que aún podría hacerse mucho más a nivel institucional y desde los poderes públicos para prestigiar las enseñanzas de FP, partiendo del gran esfuerzo realizado en los últimos años. En síntesis, las enseñanzas de FP han comenzado a andar por el camino correcto, pero aún necesitan continuar en esta línea e incluso acelerar el paso para poder llegar más lejos y equipararse a los estudios universitarios.

\section{REFERENCIAS BIBLIOGRÁFICAS}

CASQUERO, A. (2006): Transición del sistema educativo al mercado laboral en España. Especial referencia a la formación profesional (Málaga, Unicaja, Analistas Económicos de Andalucía).

DE PABLO, A. (1997): La nueva formación profesional: dificultades de una construcción, Revista Española de Investigaciones Sociológicas (REIS), 77-78, pp.137-161.

HOMS, O (2008): La formación profesional en España. Hacia la sociedad del conocimiento (Barcelona, Fundación La Caixa).

LEY de Formación Profesional Industrial de 20 de julio de 1955 (GAZETA).

LEY 14/1970, de 4 de agosto, General de Educación y Financiamiento de La Reforma Educativa (BOE de seis de agosto de 1970).

LEY 1/1990 de 3 de octubre de 1990, de Ordenación General del Sistema Educativo (LOGSE) Ley 1/1990 de 3 de Octubre (BOE de 4 de Octubre de 1.990).

II PROGRAMA NACIONAL DE FORMACIÓN PROFESIONAL (1998), aprobado por el Consejo de Ministros en su reunión del día 13 de marzo de 1998.

LEY ORGÁNICA 5/2002, de 19 de junio de las Cualificaciones y de la Formación Profesional (BOE núm. 147, de 20 de junio de 2002).

LEY ORGÁNICA 2/2006, de 3 de mayo, de Educación (BOE, núm. 106, de 4 de mayo de 2006).

MERINO, R. (2003): Luces y sombras de la reforma de la formación profesional: la visión de los profesores de enseñanza secundaria, Aula Abierta, 82, pp.5-26. 
ORDEN de 24 de septiembre de 2009, conjunta de las Consejerías de Educación y de Empleo, por la que se regula la convocatoria de un procedimiento de evaluación y acreditación de competencias profesionales adquiridas a través de la experiencia laboral o de vías no formales de formación (BOJA núm. 192 de 30 de septiembre de 2009).

PARELLADA, M. (Dir.) (2014): Informe CYD2013. (Barcelona: Fundación Conocimiento y Desarrollo).

PEREYRA, M. A., GONZÁlEZ FARACO, J. C., LUZÓN, A. y TORRES, M. (2009): Social Change and Configurations of Rhetoric: Schooling and Social ExclusionInclusion in Educational Reform in Contemporary Spain, en R. COWEN y A.M. K.AZAMIAS (Eds.) International Handbook of Comparative Education, pp. 217238 (Londres, Springer)

PÉREZ ESPARRELLS, C. (2001): La Formación Profesional y el Sistema Nacional de Cualificaciones: una clave de futuro, Revista del Ministerio de Trabajo y Asuntos Sociales, 3, pp.91-113.

QUINTANA, J. (Dir.) (2008): Crecimiento y Productividad. 50 propuestas para mejorar la productividad española (Barcelona-Madrid, IEF).

RUBIO ARRIBAS, F. J. (2009): Aspectos sociológicos del desempleo/paro y de la precariedad laboral, Nómadas: revista crítica de ciencias sociales y jurídicas, 24 , pp. 55-64.

\section{PROFESIOGRAFÍA}

\section{Enrique García Jiménez}

Profesor en el Departamento de Ciencias Humanas y Sociales, Centro de Magisterio "La Inmaculada". Universidad de Granada. Datos de contacto: Email: enriquegj@eulainmaculada.com

\section{Rocío Lorente García}

Profesora del Departamento de Pedagogía. Facultad de Humanidades y Ciencias de la Educación. Universidad de Jaén. Datos de contacto: Email: rlorente@ ujaen.es

Fecha de recepción: 16 de marzo de 2015.

Fecha de aceptación: 10 de septiembre de 2015. 